\title{
Automatic Vehicle Tracking System Based on Fixed Thresholding and Histogram Based Edge Processing
}

\author{
N. Shobha Rani, Neethu O. P., Nila Ponnath \\ Department of Computer Science, Amrita Vishwa Vidyapeetham \\ Mysore Campus, Mysore-570026, Karnataka, India
}

\begin{abstract}
Article Info
Article history:

Received Mar 19, 2015

Revised Apr 30, 2015

Accepted May 25, 2015

\section{Keyword:}

Character recognition

Edge Detection

Fixed points thresholding

Template matching

Vehicle license plate extraction

ABSTRACT

Automatic detection, extraction and recognition of vehicle number plate region in traffic control systems is one of the prominent application in Computer vision. The drastic increase in number of vehicles in the current generation greatly increases the complexity in tracking the vehicles through the human visual system, manual procedure of controlling traffic and enforcement of various laws and rules is not sufficient for smooth control of traffic. This urges the need for development of technology that can automate this process. This paper mainly focuses on the development of an automatic number plate extraction and recognition algorithm by incorporating constructs like edge detection, horizontal and vertical edge processing using fixed threshold technique. The extracted number plate region is again processed using template matching algorithm for the recognition of the characters embossed on the number plate with respect to every individual piece of number plate. The algorithm developed has achieved an accuracy of around $100 \%$ and works for both front and rear images of the car.
\end{abstract}

Copyright (c) 2015 Institute of Advanced Engineering and Science. All rights reserved.

\section{Corresponding Author:}

N. Shobha Rani, Department of Computer Science, Amrits Vishwa Vidyapeetham, Mysore Campus, Mysore, 570026, Karnataka, India. Email: n.shoba1985@gmail.com

\section{INTRODUCTION}

License Plate Extraction and Recognition systems are used to track and monitor the moving vehicles by automatically extracting the number plates. Automatic license plate recognition become very important in the current technology generation because of the unlimited increase of cars and transportation systems which causes difficulty in tracking vehicles for the purpose of parking system, traffic management system and law enforcement especially at state borders etc. The drastic increase in number of vehicles in last couple of decades really complicated the job of tracking the vehicles manually by authorities of traffic control and management. The manual method for controlling traffic and enforcement of laws is not sufficient enough to manage the traffic especially in metropolitan cities.

An automated system is necessary to identify vehicles and fetch the vehicles information since the system plays an important role in detecting security threat by identifying the individual owning the vehicle. This provides much scope for tracking the criminals who are trespassing the traffic rules and causing harm to other individuals in the society. The applications of automated traffic control systems also include tracking of stolen/suspicious vehicles i.e., system which is deployed on the road side can perform a match between the passing cars and the black list which contains a list of stolen cars or unpaid fines. The 'black list' can be updated in real time and provide immediate alarm or siren to the police force, tolling and border control i.e., the border crossings will be monitored by the system which makes use of the car numbers registered in the entry or exits to the country. It reduces the time and helps to calculate the travel fee in toll road and access 
control and parking i.e., This system will be deployed on the gate of a secured area so that, the gate automatically monitors the vehicles number plates and opens only for authorized members. The automation of all the above applications assists in resolving many conflicts that may incur during the envision of the vehicle tracking. The tracing of suspicious vehicles will be done very economically without consumption of lots of man power.

An efficient and reliable system for license plate extraction and recognition is to be introduced to solve the challenges in manual method tracking the vehicles. This makes us to motivate for the research on development of automated technology that quenches the multiple needs in traffic control and management.

\section{RELATED WORK}

Number plate extraction and recognition had undergone numerous experimentations by various researchers. Many approaches were implemented and had undergone many changes in the view of improvising accuracy in license plate extraction; some of the approaches developed by researchers are reviewed below.

Sarbjit Kaur et. al [1] has proposed an algorithm for automatic number detection and recognition using morphological operations for pre-processing, Sobel operator for vertical edge detection, connected component analysis for segmentation and recognition. The algorithm have attained a over all accuracy of around 97\%. Serkan Ozbay et. al. [2] had propped a simple algorithm using edge detection algorithms, smearing algorithms and template matching based recognition approach for character recognition. The algorithm is specifically designed for the recognition requirements of Turkish license plates and had achieved an overall accuracy of around 97\%. D.G. Bailey et. al [3] had designed a modular structure interface for evaluation and comparison analysis of various for number plate recognition algorithms. Johnson et al [4] had devised an algorithm for number plate recognition by employing optical character recognition techniques. Naikur Bharatkumar Gohil et.al [5] has propped an approach on car license plate detection using histogram based approach, it processes each frame individually and provides the co-ordinates of location with maximum probability of having a number plate. Rinku solanki et.al [6] has proposed a method of automatic license plate recognition, in which license plate is extracted based on some features like color, the boundary, or the existence of the characters. In the license plate segmentation stage, the characters are extracted by projecting their color information, by labeling them, or by matching their positions with template. Kumar Parasuraman et.al [7] has contributed an approach on Indian vehicle license plate extraction and character segmentation based on the morphological algorithms and connected components analysis. Sandra Sivanandan et.al [8] has contributed a methodology on automatic vehicle identification through edge detection and morphological operations, a Scan line algorithm for segmentation and recognition of segmented characters. Chetan Sharma et.al [9] and has devised a method on Indian Vehicle license Plate Extraction using histogram equalization method, morphological operations and edge Detection techniques. Sheetal Mithun Kawade et.al [10] has contributed an approach of a real time vehicle license plate recognition by employing an appropriate threshold technique for segmentation and template matching technique for recognition. Pratishtha Gupta et.al [11] has developed an automated system using SIMULINK model in Mat lab which extracts the number plate and recognize alphanumeric characters and recognition is performed using template matching. Ronak P. Patel et.al [12] has proposed an approach on automatic licenses plate recognition using morphological operations and edge detection techniques. A.Akoum et.al [13] has proposed a new approach for detection and Identification of vehicle number by combining features of horizontal gradients and method symmetry.

The list of experimentations on extraction and recognition of vehicle license plate are very wide. It is clearly noticeable that many methodologies are devised using techniques of pre-processing like morphological operations, Canny edge detection and histogram matching etc. The scientific observations of existing methodology with the proposed datasets employing canny edge detection and sobel dge edge detection are as follows.

Both the Sobel edge detector and Canny edge detector uses a pair of 3x3 convolution matrices for identifying the intensity discontinuities in the images. The very important prospect of sobel edge detctor is, it is dedicated for evaluation of vertical edges and horizontal edges. It is very efficient compared to any edge detction operator when it comes to identification of horizontal or vertical edges, since canny edge detection to extract useful structural information from different vision objects and dramatically reduce the amount of data to be processed.

The reduction of number of smaller edges is not negligible in case of sobel edge detection and the smaller or minute edges are very important in the researches related to the extraction of number plate region because the characters embossed on the number plate are composed of very thin or minute edges. More over the major focus of the experimentation is to extract the number plate region form a car image and recognize the various characters embosed on it. Thus the current experimentation has considered the Sobel edge 
detection as ideal for detection of intensity discontinuities in the images. The Figure 1(a) and Figure 1(b) shows the results of sobel and canny edge detection on the input images imbibed. The proposed methodology contributes a novel algorithm which makes use of a Sobel edge detection method and a fixed threshold technique for the detection of license plate region and an automatic image cropping method for the extraction of license plate region.

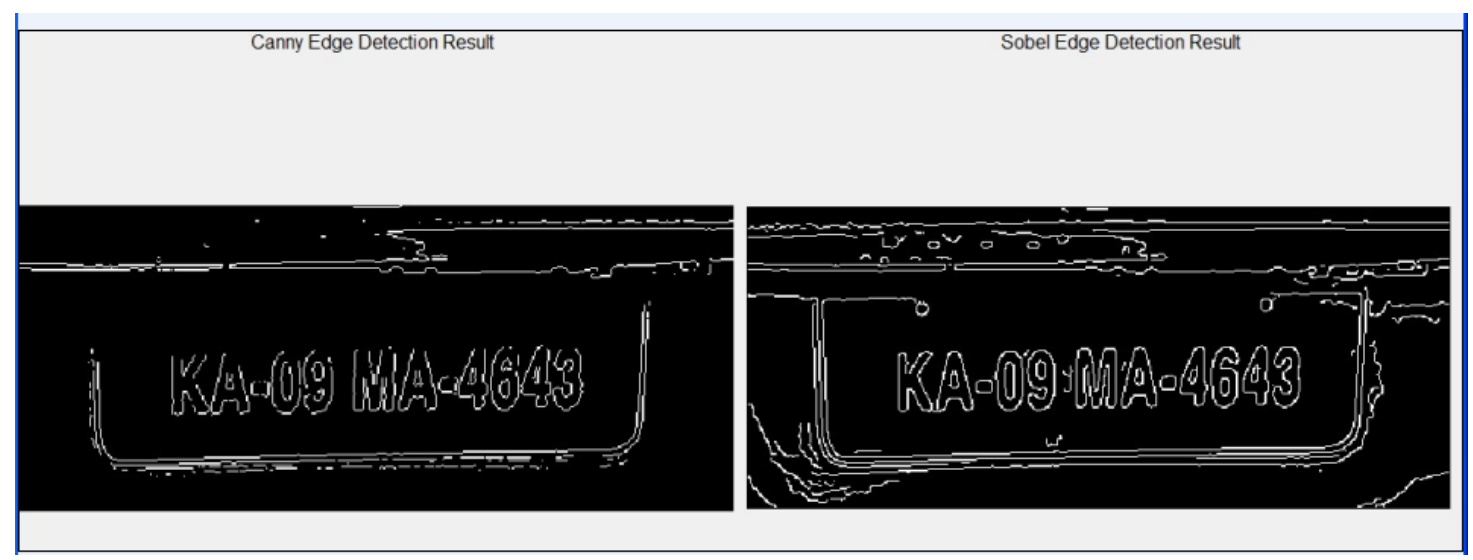

Figure 1(a). Results of Canny and Sobel edge detection
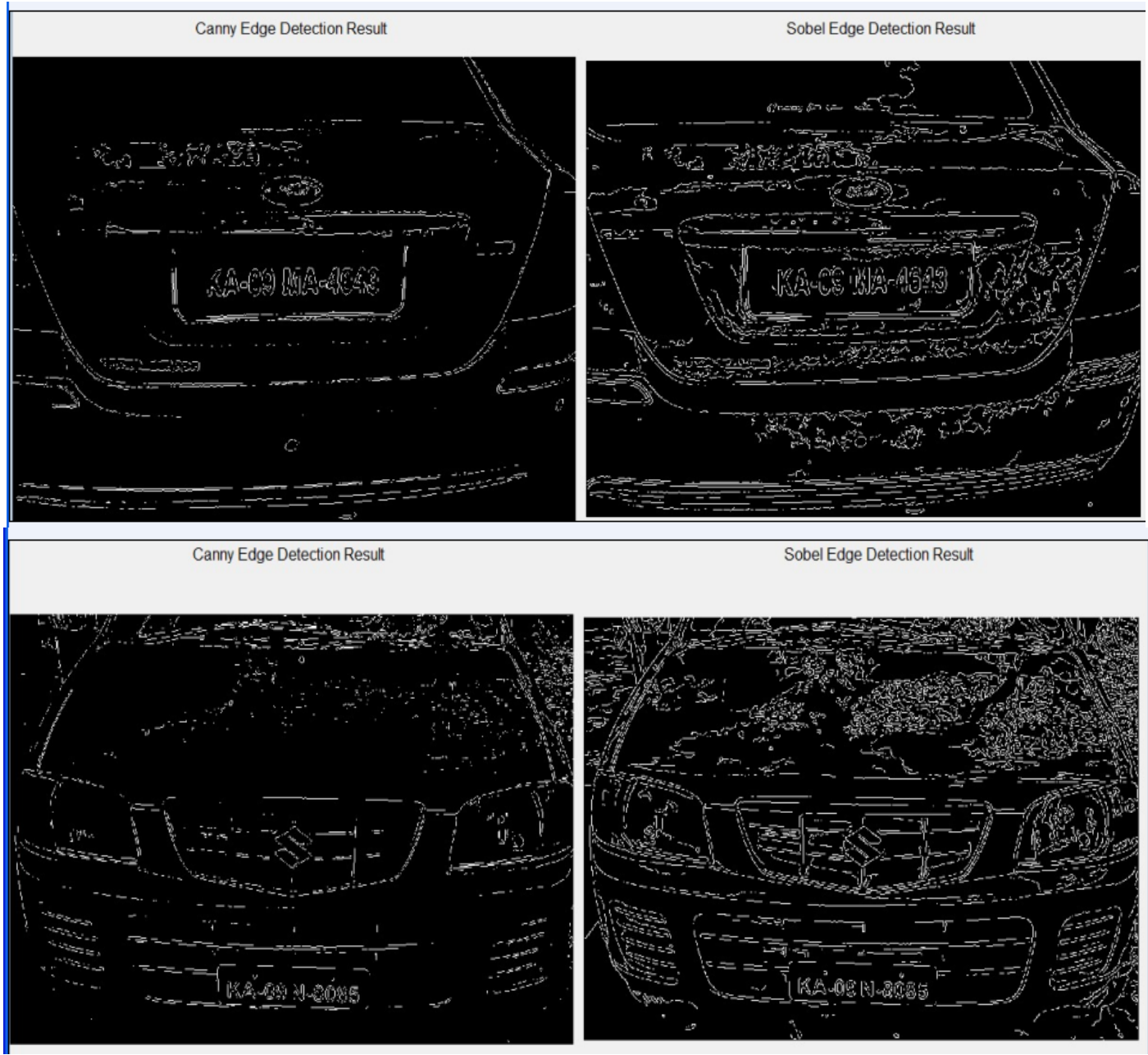

Figure 1(b). The very smaller details are not retained in the left images where as in right image the finer edge details are preserved as a result of Sobel operators. 


\section{RESEARCH METHOD}

In the proposed methodology the extraction and recognition of number plate region on car images is accomplished in three stages. In stage 1, pre-processing of the input image is performed to convert the input image into an enhanced or noise-free gray scale image. The extraction of number plate regions is achieved through histogram based horizontal and vertical edge processing using a fixed threshold technique in stage 2 to identify the probable regions of number plate and the stage 3 encompasses the segmentation and recognition of characters embossed on the number plate region by applying bouding box method and template matching algorithm for recognition. The user interface designed for the proposed system is as depicted in the Figure 2.

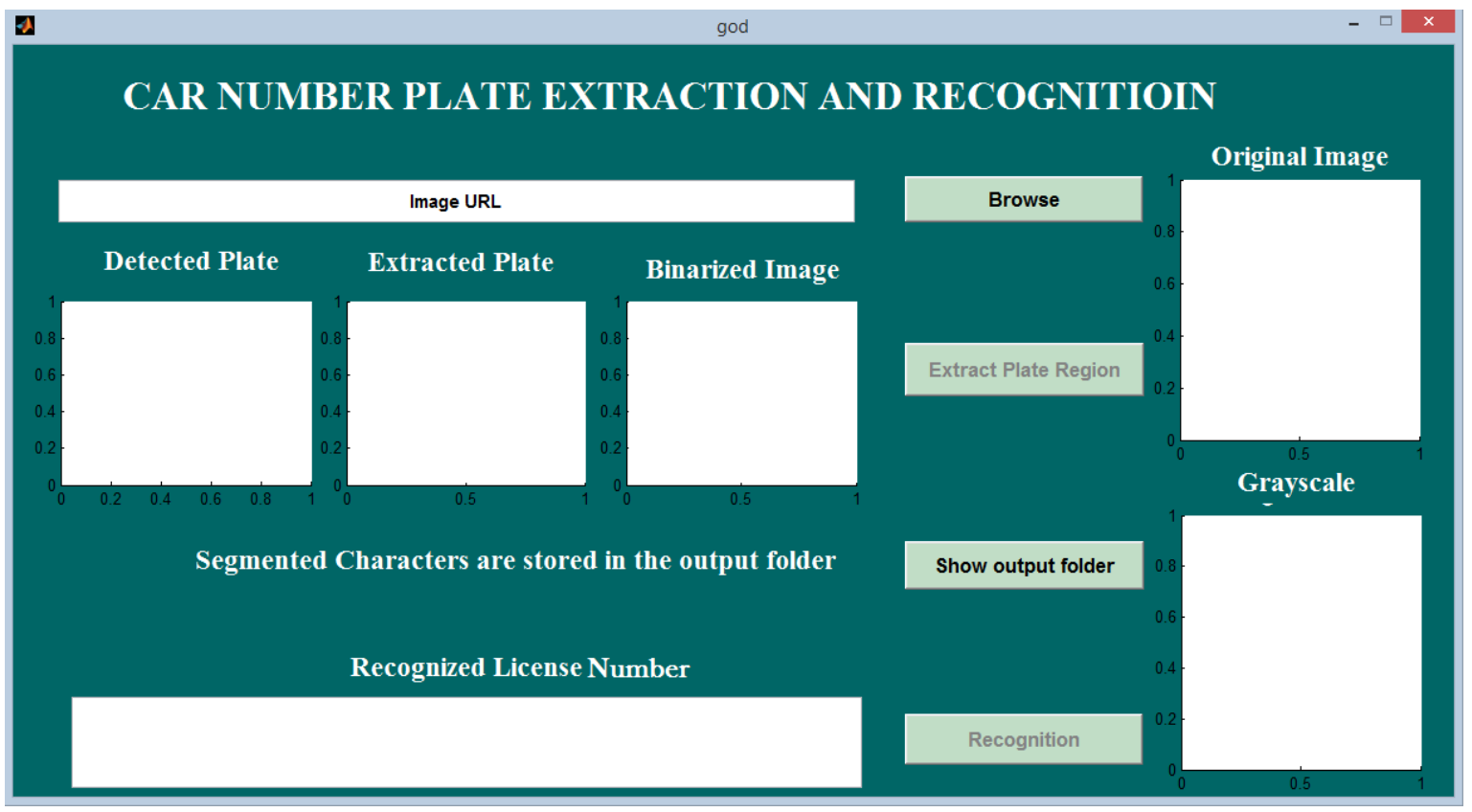

Figure 2. Graphical user interface for automatic number plate extraction and recognition

\subsection{Pre-Processing:}

The pre-processing is a pre-requisite operation for any of the image processing system to convert the input images into the form suitable for succeeding stages of processing. In the proposed system preprocessing encompasses the stage 1 of the automatic number plate extraction and recognition. Initially a RGB image is considered as input to the proposed system which will be transformed to a gray scale image. Further Gaussian filtering [2] is applied to eliminate the noise from the image and highlight the high frequency components in the image, since images captured in real time may consists of lots of back ground noise and unbalanced illumination artifacts. Morphological operation is applied on the image to connect the broken edge and the gradient operations [4] are performed to thicken the edges of the image. First order derivative mask, Sobel is applied to highlight the edges oriented at horizontal and vertical directions. The Figure 3 shows the input datasets used for automatic number plate extraction and recognition. The figure 4 provides the output of pre-processing.

\subsection{Detection of license plate region}

The stage 2 of the proposed methodology employs histogram based processing for detection of horizontal and vertical edges through which the probable number plate region can be detected. Region with the maximum histogram value is considered as the most probable candidate for number plate, since in the pre-processed gray scale image horizontal and vertical edge are highlighted and which will have dominating continuous high frequencies defined than any other edges in the vehicle image. The vertical and horizontal histogram of the sharpened image is obtained and which is has depicted in the Figure 5.

All the regions in the image are processed using row-wise and column-wise to find a common region having maximum horizontal and vertical histogram value. The common region is the region where the horizontal and vertical histogram frequencies matches or intersects with one another. 


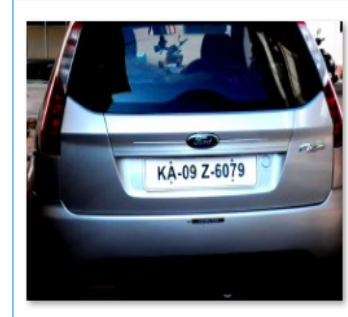

$20141230 \_094318$

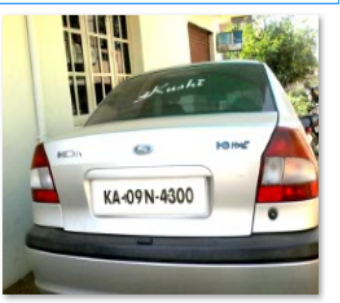

IMG-20150203-WA0013

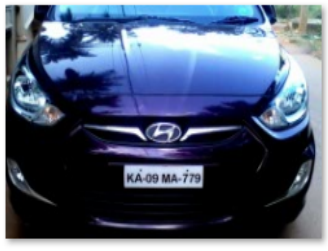

20141231_164817

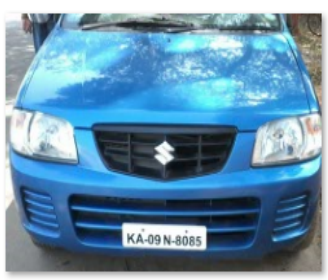

IMG-20150203-WA0014

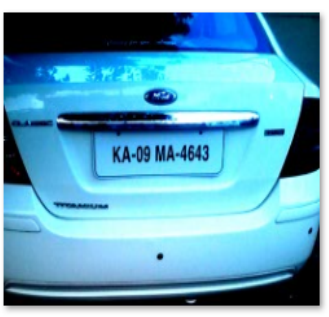

20141231_170021

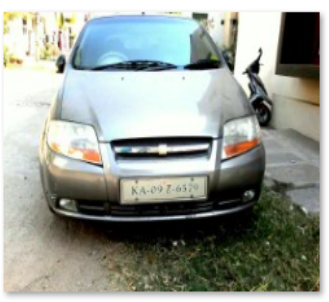

IMG-20150203-WA0015

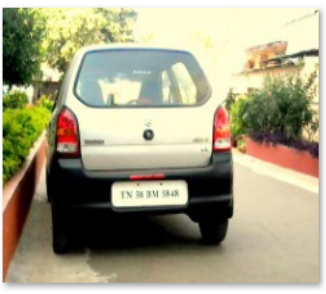

IMG-20150203-WA0012

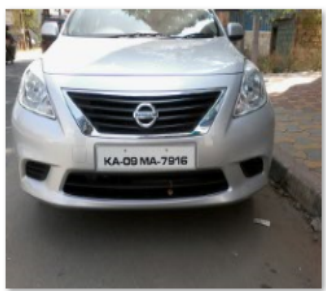

IMG-20150203-WA0016

Figure 3. Input datasets for automatic number plate extraction and recognition

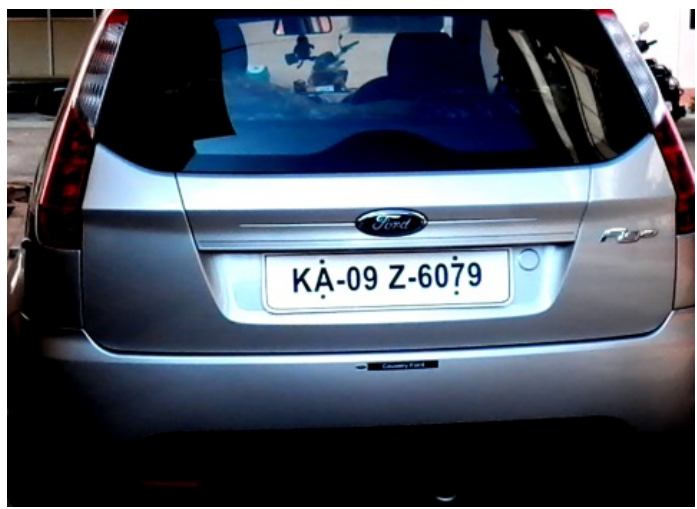

(a) Original Image

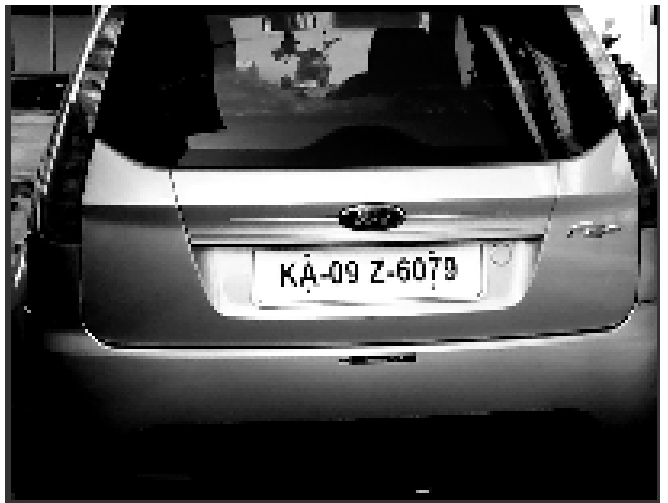

(b) Gray scale Image

Figure 4. Output of pre-processing stage

The common region which is obtained using histogram matching [5] technique is considered as region with highest probability of containing a license plate number.

Further the extraction of common region in the vehicle image is accomplished through automatic image cropping using a fixed range method. Fixed Range [14] is a method by which we determine the range block which contains the span of convergence regions with respect to both the horizontal and vertical histograms by appending with span of additional rows and columns in top, bottom, left and right of convergence region identified with respect to a threshold value 'N1' and 'N2'.

\subsubsection{Determination of threshold value 'N1' and 'N2':}

The threshold value ' $\mathrm{N} 1$ ' and ' $\mathrm{N} 2$ ' used in the fixed range method is a value which is determined by applying differences operation between the pixel intensity values available within the convergence region of the image. Consider a pixel p1, p2, p3...pn in the convergence region of a gray scale image with respect to a row or column. The difference between the pixel pi - pi+1 where $i=1,2,3 \ldots n$ is computed with respect to each pair of adjacent pixels in the convergence region and stored in a row vector and similarly the column wise intensity differences are also calculated and stored in a column vector. The minimum difference value $\operatorname{Min}(r)$ and maximum difference value $\operatorname{Max}(r)$ is determined from the row vector and Min(c) and Max(c) are determined from the column vector. The minimum and maximum differences obtained from the row and column vector can be accorded as the minimum fixed threshold and maximum fixed threshold. The threshold value 'N1' and 'N2' is empirically given by average of $\operatorname{Min}(r), \operatorname{Max}(r)$, Min(c) and $\operatorname{Max}(\mathrm{c})$ with respect to its row and column. 


$$
\begin{aligned}
& \text { i.e., } N 1=\frac{\operatorname{Min}(r)+\operatorname{Min}(c)}{2} \\
& \text { i.e., } N 2=\frac{\operatorname{Max}(r)+\operatorname{Max}(c)}{2}
\end{aligned}
$$

The Figure 6 shows the snapshot of the frequencies vailable within the convergence region of the image.

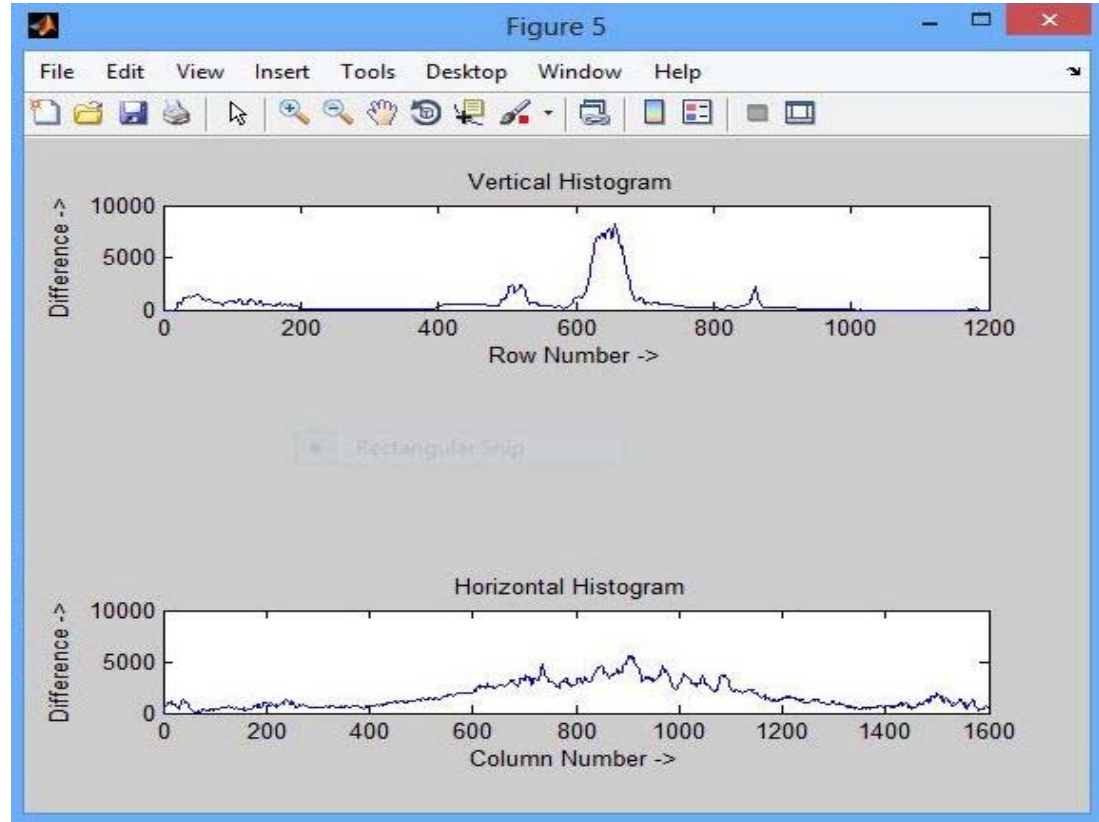

Figure 5. Horizontal and Vertical histograms of Figure 4

$\begin{array}{rrrrrrrrrrrrrrrr}213 & 215 & 216 & 216 & 214 & 206 & 194 & 158 & 128 & 108 & 108 & 113 & 115 & 118 & 117 & 116 \\ 210 & 213 & 216 & 218 & 216 & 210 & 195 & 164 & 130 & 109 & 108 & 112 & 117 & 120 & 119 & 117 \\ 212 & 214 & 217 & 219 & 216 & 212 & 194 & 171 & 133 & 110 & 108 & 110 & 117 & 120 & 120 & 117 \\ 214 & 215 & 216 & 218 & 214 & 213 & 195 & 180 & 141 & 115 & 111 & 109 & 115 & 118 & 120 & 117 \\ 212 & 213 & 214 & 217 & 214 & 215 & 200 & 187 & 152 & 125 & 115 & 110 & 113 & 119 & 122 & 121 \\ 211 & 213 & 214 & 217 & 216 & 216 & 205 & 189 & 162 & 133 & 116 & 110 & 109 & 119 & 123 & 124 \\ 212 & 213 & 214 & 217 & 218 & 217 & 212 & 190 & 169 & 137 & 113 & 106 & 102 & 116 & 120 & 123 \\ 212 & 212 & 213 & 216 & 218 & 218 & 219 & 194 & 172 & 139 & 110 & 102 & 96 & 114 & 117 & 121 \\ 212 & 215 & 211 & 213 & 218 & 221 & 217 & 199 & 177 & 143 & 112 & 101 & 102 & 110 & 117 & 120 \\ 211 & 213 & 213 & 215 & 219 & 221 & 215 & 202 & 177 & 150 & 114 & 99 & 106 & 109 & 119 & 121 \\ 212 & 211 & 214 & 216 & 219 & 220 & 214 & 207 & 178 & 158 & 118 & 97 & 109 & 107 & 118 & 119 \\ 214 & 211 & 215 & 214 & 216 & 220 & 215 & 211 & 184 & 164 & 126 & 100 & 107 & 109 & 117 & 118 \\ 213 & 211 & 215 & 213 & 215 & 221 & 216 & 212 & 193 & 166 & 135 & 108 & 104 & 113 & 116 & 118 \\ 211 & 211 & 214 & 215 & 217 & 221 & 218 & 212 & 200 & 168 & 142 & 115 & 103 & 114 & 116 & 118 \\ 211 & 211 & 213 & 217 & 219 & 219 & 219 & 214 & 205 & 173 & 146 & 119 & 107 & 109 & 115 & 117 \\ 212 & 212 & 211 & 218 & 219 & 217 & 220 & 218 & 209 & 179 & 149 & 121 & 112 & 106 & 114 & 116 \\ 213 & 212 & 214 & 214 & 219 & 219 & 219 & 216 & 210 & 184 & 153 & 127 & 110 & 104 & 109 & 117 \\ 212 & 212 & 214 & 214 & 219 & 219 & 220 & 216 & 211 & 193 & 158 & 133 & 114 & 103 & 110 & 115 \\ 212 & 211 & 213 & 213 & 218 & 219 & 210 & 216 & 211 & 202 & 164 & 139 & 118 & 101 & 109 & 111 \\ 211 & 210 & 211 & 211 & 216 & 218 & 219 & 216 & 211 & 206 & 173 & 145 & 122 & 104 & 107 & 109 \\ 210 & 219 & 210 & 2199 & 214 & 216 & 218 & 216 & 214 & 208 & 184 & 151 & 126 & 109 & 103 & 107 \\ 210 & 219 & 210 & 218 & 213 & 214 & 217 & 216 & 215 & 208 & 194 & 158 & 128 & 112 & 99 & 105 \\ 210 & 210 & 212 & 210 & 213 & 214 & 217 & 216 & 216 & 210 & 202 & 167 & 134 & 117 & 103 & 109 \\ 210 & 211 & 214 & 212 & 215 & 215 & 217 & 217 & 218 & 213 & 208 & 177 & 141 & 123 & 112 & 117\end{array}$

Figure 6. Frequencies in the convergence region of image 


\subsection{Extraction of license plate region}

Initially the gray scale image is processed horizontally to determine the horizontal edges by traversing each and every row by calculating the difference every two adjacent pixels and if difference between pixel intensities does not falls within the threshold N1 and N2 then, those pixels will be marked with ' 0 ' intensity(black). This processed will be continued until all the rows are exhausted in the image.

To determine the vertical edges in the probable license plate region, each column is traversed and column wise differences are computed, if the difference between any two adjacent pixels does not fall within the threshold ' $\mathrm{N} 1$ ' and 'N2' then those pixels are marked with ' 0 ' intensity(black). This process will be continued till all the columns in the image exhausts. Further the resultant image is smoothened by applying a smoothing filter [6] to eliminate the distinctly marked pixels which are considered as unwanted regions. The Figure 7 depicts the results obtained after horizontal and vertical edge processing using fixed threshold method.

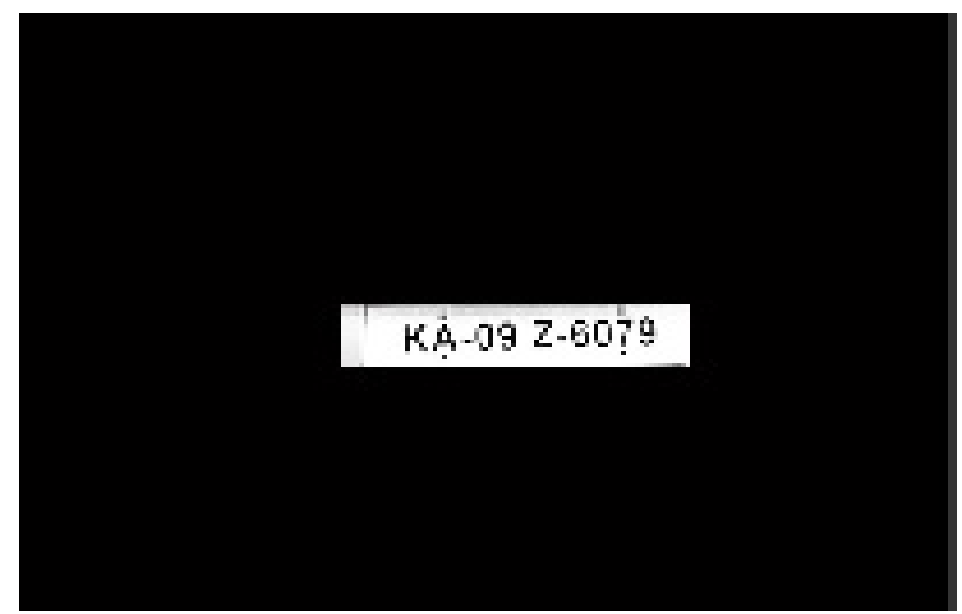

Figure 7. Output of Horizontal and vertical edge processing using fixed threshold

Our next step is extraction of license plate region from the resultant image. In the proposed system, we first crop the image horizontally and then vertically. In horizontal cropping we process the image matrix columnwise and compare its horizontal histogram value with the common region frequency values. If certain value in the horizontal histogram is more than threshold, we make it as our starting point for cropping and continue until threshold value we find less than that is our end point. In this process we encounter many areas which have value more than threshold. So we store are starting and end point in the matrix and compare width of each area; width is calculated difference of start and end points. Then we crop image horizontally by using the start and end point. This new horizontally cropped image is processed for vertical cropping. In vertical cropping we use the same threshold comparison method, but the only difference is that this time we process image matrix row-wise and compare threshold value with vertical histogram values. Again we get set vertical start and end point, we find that set which map largest height and crop the image. After vertical and horizontal cropping we get exact area of number plate from original image in RGB format. The extracted license plate region is as shown in Figure 8.

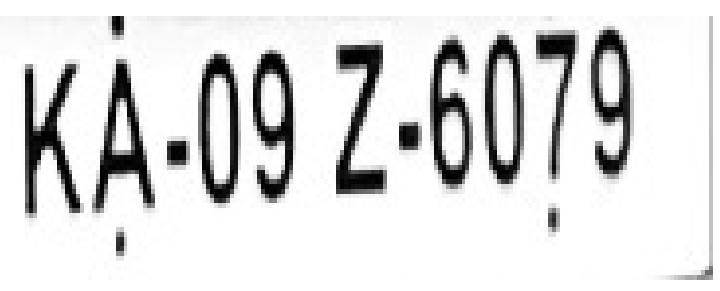

Figure 8. Extracted number plate region 


\subsection{Recognition of License Plate}

The extracted number plate is processed again the stage 3 to recognize the characters embossed on the license plate. The extracted number plate image from stage 2 is binarized [2] and then segmentation of the characters in the number plate is performed using vertical projection profiles [3] and bounding box approach [5]. The Figure 9 and figure 10 shows the outputs of binarization and segmentation.

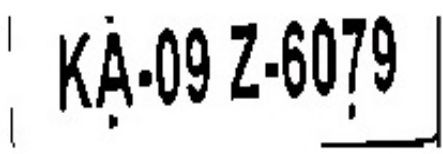

Figure 9. Binarized image

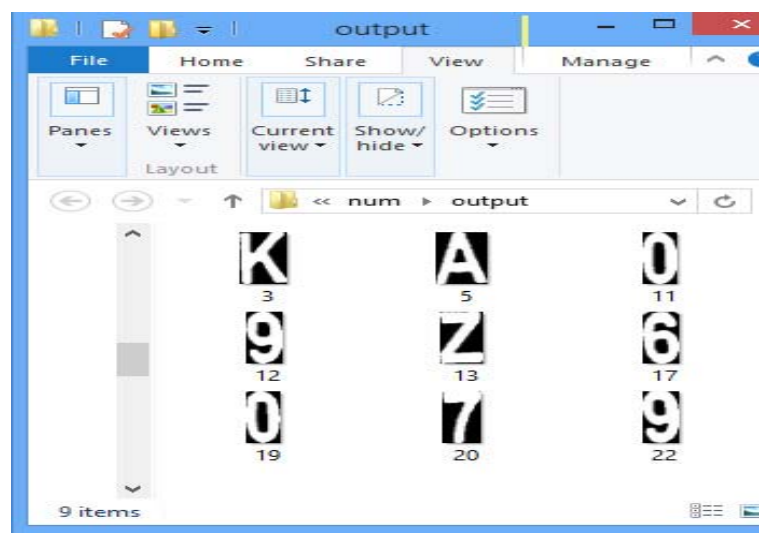

Figure 10. Segmented characters displayed in output folder

Further template matching [11] based classification is used for recognition of individual alphanumeric character. In template based algorithm, segmented image is compared with the images which are stored in database. During classification, the test image for which the correlation coefficient for template image is maximum, that image is considered as a best match. The each and every character classified is post processed and displayed in editable format in a notepad. The Figure 11(a), Figure 11(b), Figure 11(c) provides the recognition results and Figure 12 provides the recognized characters in a log file.

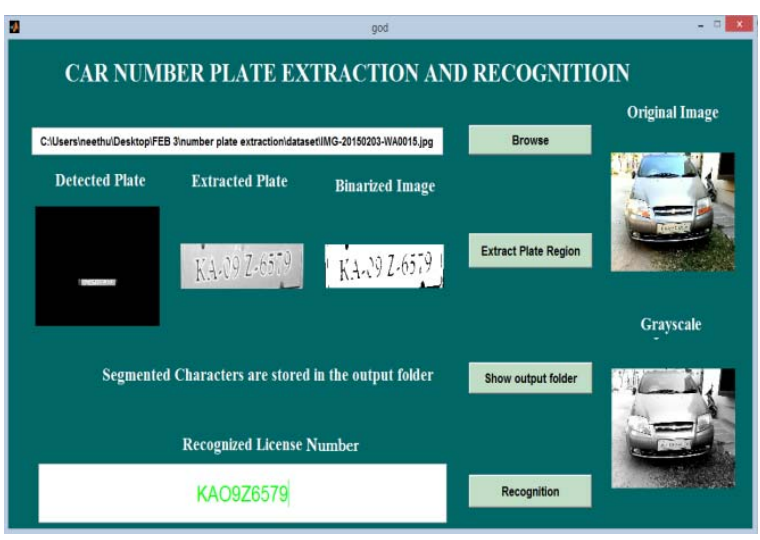

Figure 11. (a) Output of GUI interface

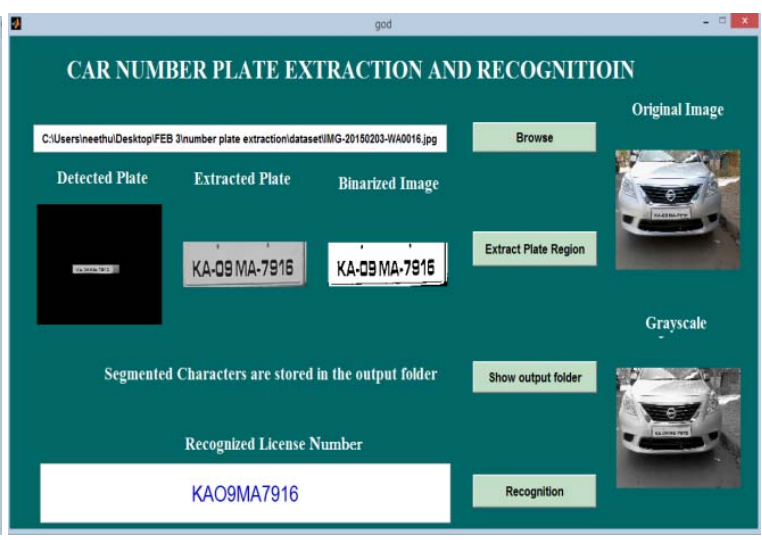

Figure 11. (b) Output of GUI interface 


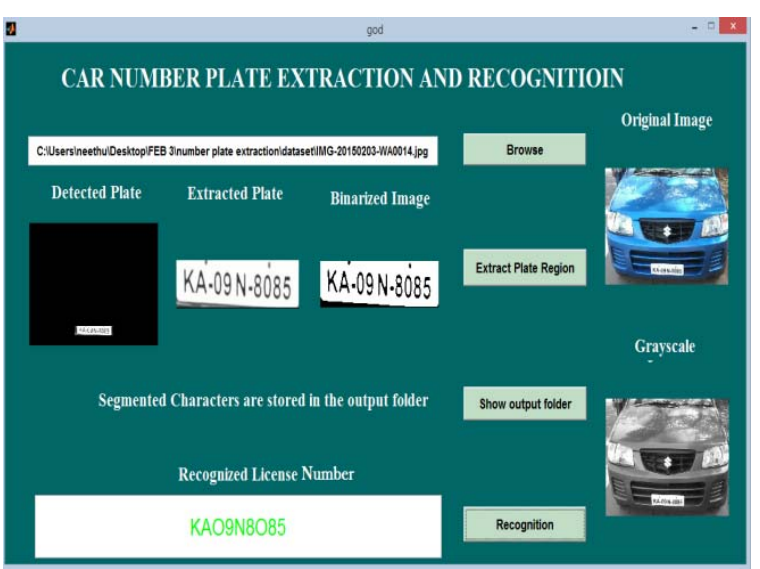

Figure 11. (c) Output of GUI interface

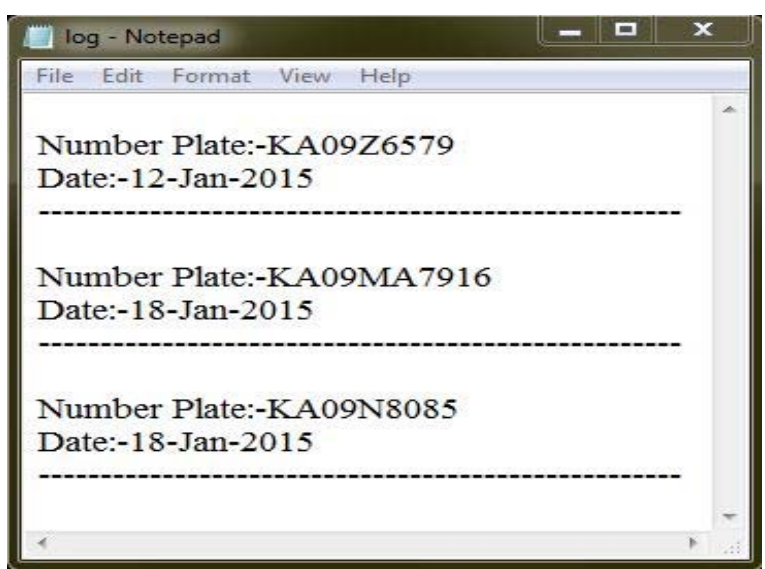

Figure 12. Log file of recognized characters

\section{RESULTS AND ANALYSIS}

The proposed algorithm was tested with 65 input images having different resolutions and captured at different illumination conditions. The images contained vehicles of different colors and includes front and rear view of the car. The front images of car tested are around 45 and rear images of about 20 are tested and $100 \%$ accuracy is obtained for the front images of car, where as rear images has given an accuracy of $98 \%$ in recognition of number plate. The algorithm is flexible to work with all types of images. The accuracy in the proposed system completely depends upon the histogram processing in identification of convergence region with respect to both horizontal and vertical histogram. The sobel edge detection for the current experimentaion had produced very good outcomes, which as result lead towards an error free recognition process of number plate recognition.. The efficiency of the system is defined as number of test images recognized correctly to the total number of images considered for testing. The algorithm has achieved an overall accuracy of $99 \%$ with all the images tested.

\section{CONCLUSION}

The detection and extraction of license plate region of car images is one the challenging task in the process of license plate recognition. The histogram based processing for the identification of probable license plate region is core operation in the proposed system. The determination of fixed threshold N1 and N2 using adjacent pixel differences is at the heart of the proposed system using which we can extract the license plate region in the image. The algorithm can be further extended for the recognition of license plates in two wheeler vehicle images. The algorithm can also be enhanced further to recognize license plate of images captured at longer focal lengths.

\section{REFERENCES}

[1] Sarbjit Kaur, Sukhvir Kaur, "An Efficient Approach for Number Plate Extraction from Vehicles Image under Image Processing", International Journal of Computer Science and Information Technologies, Vol. 5 (3) , 2014, 2954-2959.

[2] Serkan Ozbay, Ergun Ercelebi, "Automatic Vehicle Identification by Plate Recognition", World academy of Science, Engineering and Technology International Journal of Electrical, Computer, Electronics and Communication Engineering Vol:1 No:9, 2007.

[3] D.G. Bailey, D. Irecki, B.K. Lim and L. Yang "Test bed for number plate recognition applications", Proceedings of First IEEE International Workshop on Electronic Design, Test and Applications ( DELTA'02 ), IEEE Computer Society, 2002.

[4] A.S. Johnson, B.M., "Bird, 1990, "Number-plate matching for Automatic Vehicle Identification," IEEE Colloquium on Electronic Image and Image Processing in Security and Forensic, April, 1990.

[5] Naikur Bharat Kumar Gohil, "Car license plate detection", Dharmsinh Desai University", India, 2006.

[6] Rinku Solanki, Rajesh Kumar Rai, Teena Raikwar, "The automatic license plate recognition", International journal of engineering research and applications, ISSN: 2248-9622, vol. 3, Issue 6, Nov-Dec 2013, pp 304-310.

[7] Kumar Parasuraman, P. Vasantha Kumar, "An efficient method of Indian vehicle license plate extraction and character segmentation", IEEE International conference on computational intelligence and computing research, 2010. 
[8] Sandra Sivanandan, Ashwini Dhanait, Yogita Dhepale, "Automatic vehicle identification using license plate recognition for Indian vehicles", IJCA proceedings on emerging trends in computer science and Information technology, 2012.

[9] Chetan Sharma, Amardeep Kaur, "Indian Vehicle license Plate Extraction and segmentation", International journal of Computer Science and Communication, July-December 2011, Vol. 2, pp. 593-599.

[10] Sheetal Mithun Kawade, M.M. Mukhedkar, "A Real time vehicle's license plate recognition system", International journal of Science and Engineering, Vol. 1(2), pp. 41-48.

[11] Pratishtha Gupta, G. N. Purohit, Manisha Rathore, "Number Plate extraction using Template matching technique", International journal of Computer applications, ISSN: 0975-8887, Vol. 8(3), February 2014.

[12] Ronak P Patel, Narendra M Patel, Keyur Brahmbhatt, "Automatic License Plate Recognition", International jOurnal of Computer Science and Mobile Computing, April 2013.

[13] A. Akoum, B. Daya, P. Chauvet, "A New approach for detection and identification of vehicle plate numbers", Journal of software engineering and applications, Vol. 3(2), pp. 99-108, February 2010.

[14] Edward R Doughert, "Digital Image Processing Methods", CRC Press, 12-Jan-1994 - Technology \& Engineering 504 pages.

\section{BIOGRAPHIES OF AUTHORS}

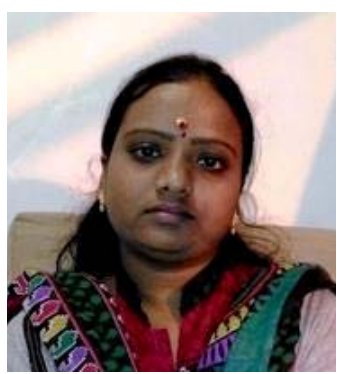

N. Shobha Rani has completed M.Sc (CS) and currently pursuing her Ph.D degree in Computer Science and technology at Maharaja Research Foundation, MIT, Mysore and working as a faculty in the department of Computer Science at Amrita Vishwa Vidyapeetham, Mysore Campus. Her area of interests is Optical character recognition, Computer vision and Document image processing.

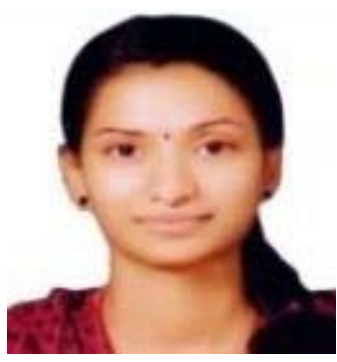

Neethu O.P. is currently pursuing her Master's degree in Computer applications (MCA) in department of Computer Science at Amrita Vishwa Vidyapeetham, Mysore Campus. Her area of interests is Computer vision and image processing.

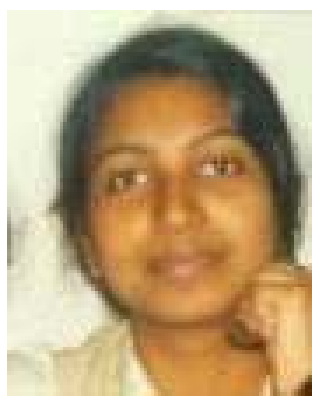

Neethu O.P. is currently pursuing her Master's degree in Computer applications (MCA) in department of Computer Science at Amrita Vishwa Vidyapeetham, Mysore Campus. Her area of interests is Computer vision and image processing. 